\title{
Structure-function interplay as signature for brain decoding and fingerprinting
}

\author{
Alessandra Griffa ${ }^{1,2}$, Enrico Amico ${ }^{2,3}$, Raphaël Liégeois ${ }^{2,3}$, Dimitri Van De Ville ${ }^{2,3,4}$, Maria \\ Giulia Preti ${ }^{2,3,4}$ \\ ${ }^{1}$ Department of Clinical Neurosciences, Division of Neurology, Geneva University Hospitals \\ and Faculty of Medicine, University of Geneva, Geneva, Switzerland \\ ${ }^{2}$ Institute of Bioengineering, Center of Neuroprosthetics, Ecole Polytechnique Fédérale De \\ Lausanne (EPFL), Geneva, Switzerland \\ ${ }^{3}$ Department of Radiology and Medical Informatics, University of Geneva (UNIGE), Geneva, \\ Switzerland \\ ${ }^{4}$ CIBM Center for Biomedical Imaging, Switzerland
}

\begin{abstract}
Brain signatures of functional activity have shown promising results in both decoding brain states; i.e., determining whether a subject is at rest or performing a given task, and fingerprinting, that is identifying individuals within a large group. Importantly, these brain signatures do not account for the underlying brain anatomy on which brain function takes place. Here, we leveraged brain structure-function coupling as a new imaging-based biomarker to characterize tasks and individuals. We used multimodal magnetic resonance imaging and the recently introduced Structural-Decoupling Index (SDI) to quantify regional structure-function interplay in 100 healthy volunteers from the Human Connectome Project, both during rest and seven different tasks. SDI allowed accurate classifications for both decoding and fingerprinting, outperforming functional signatures. Further, SDI profiles in resting-state correlated with individual cognitive traits. These results show that brain structurefunction interplay contains unique information which provides a new class of signatures of brain organization and cognition.
\end{abstract}

\section{Introduction}

The existence of brain signatures based on functional magnetic resonance imaging (fMRI), meaning specific features uniquely characterizing one's brain or a specific task-related state, has emerged in the last two decades. On the one hand, the application of pattern recognition techniques to neuroimaging data proved the capability of fMRI to decode task-specific brain activity ${ }^{1-5}$. Significant progress in this direction was made by the recent advent of deep learning $1,3,5$, even if it remains nontrivial to interpret the biological meaning of the learned features. On the other hand, similarly to a fingerprint, fMRI-based features can accurately identify individuals from a large group ${ }^{6-9}$. In a seminal paper from Finn and colleagues ${ }^{8}$, functional connectivity (FC) profiles were used to successfully classify subjects across restingstate test-retest sessions, and even between task and rest conditions. The fronto-parietal network emerged as the main contributor to subject discrimination, and was shown to predict individual cognitive behavior (level of fluid intelligence). In addition to functional activity, brain anatomical features, such as cortical morphology and white-matter structural connectivity, were also proven useful for brain fingerprinting ${ }^{10-14}$. 
In this context, a still unexplored brain feature, which could offer new insights into brain decoding and fingerprinting, is the structure-function interplay; i.e., how brain functional activity relates to the underlying structural connectivity architecture, measured with diffusion-weighted MRI. Early attempts to investigate structure-function relationships in the brain spanned from simple approaches, such as linear analyses ${ }^{15-18}$, to more complex ones like whole brain computational and communication models ${ }^{19-22}$. More recently, graph signal processing provided a novel framework for a combined structure-function analysis ${ }^{23-25}$. Within this setting, Preti et al. quantified the degree of structure-function dependency for each brain region, by means of the newly introduced structural decoupling index (SDI) ${ }^{25}$. During resting-state in healthy subjects, structure-function dependencies showed a very characteristic and behaviorally relevant spatial distribution, spanning from lower-order functional areas such as visual and somatosensory cortices, with function highly aligned to the structure underneath, to higher-order ones, with function more independent from the structure. However, the extent to which this configuration changes in different task-related states, or in different subjects, still remains unexplored. In particular: do structure-function dependency patterns represent a signature of a particular task-related state? And can they act as a brain fingerprint uniquely identifying individuals?

To answer these open questions, we analyzed 100 unrelated healthy subjects from the Human Connectome Project (HCP) ${ }^{26}$, and assessed their structure-function interplay profile through SDI, during resting-state and while engaged in seven different tasks. By only using structurefunction dependency values, we then attempted to classify different tasks and individuals, and in both cases, the classification showed a very high accuracy in various cross-validation settings, and a better performance than the one obtained with functional connectivity. Two specific networks including regions that are key to either brain decoding or brain fingerprinting emerged. Structure-function dependencies in resting-state were then shown to correlate with individual cognitive traits (fluid intelligence, spatial orientation, sustained visual attention and episodic memory).

\section{Results}

\section{Brain decoding and fingerprinting networks}

The structure-function interplay assessed with structural-decoupling index yielded a brain pattern of regional values (360 cortical regions of Glasser parcellation and 19 subcortical areas) for each subject and acquisition (Resting-state and 7 tasks: Emotion, Gambling, Language, Motor, Relational, Social, Working-Memory; each acquired with 2 phase encoding directions). Average SDI profiles across subjects for each state are reported in Supplementary Fig. 1.

A two-factor analysis of variance (ANOVA) assessing differences of nodal SDIs across subjects and tasks yielded two very distinct and spatially specific brain patterns (Fig. 1), characterized by a significant effect for either decoding (task-effect) or fingerprinting (subjecteffect), respectively (F-test, only nodes with significant F-values are visualized as non-zeros in Fig. 1, with $p<.05$, Bonferroni-corrected for the number of brain regions). The brain decoding pattern (Fig. 1A) clearly involves more prominently regions of task-related networks, in particular visual and somatomotor networks. On the contrary, the brain fingerprinting pattern (Fig. 1B) was spatially more spread, but concerned mainly the posterior parietal cortex, 
including fronto-parietal regions, consistently with what was found previously ${ }^{8}$, but also visual, somatomotor and dorsal attention networks.

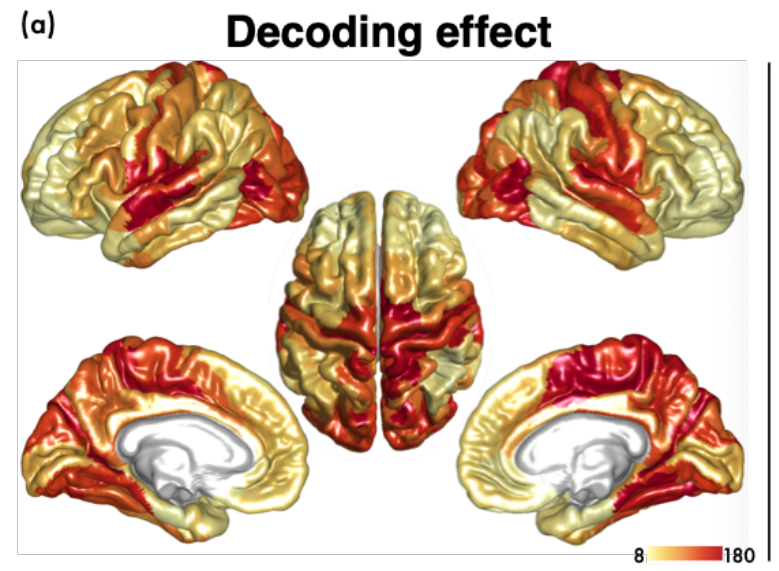

(b) Fingerprinting effect

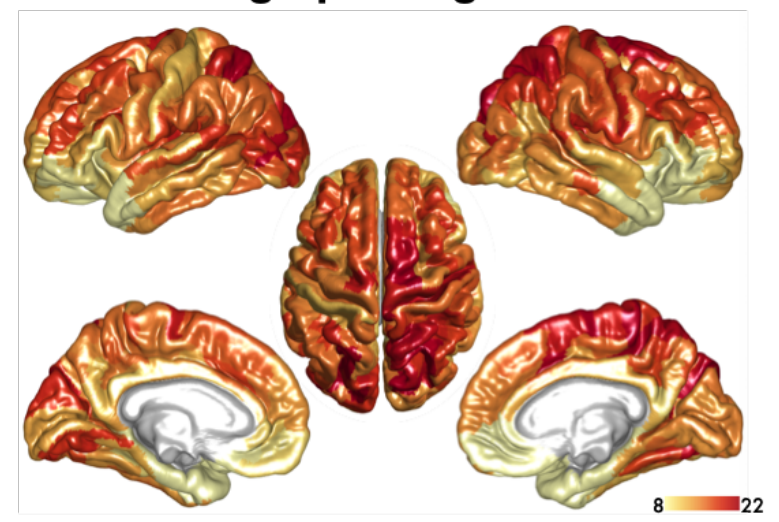

FIG. 1 - Brain networks of task (brain decoding) and subject (brain fingerprinting) main effects on structure-function dependency. Two-Factors ANOVA, significant F-values, $p<.05$ Bonferroni corrected.

\section{Structure-function interplay for brain decoding}

A Linear Discriminant Analysis (LDA) was used to classify different task-related states (restingstate and seven tasks) based on SDI values. Prior to SDI computation for this classification, task paradigms were regressed out from functional time courses to cancel out any paradigmimposed effect, aiming at keeping only differences due to the specific task-related states, and not directly driven by the paradigm characteristics. The same operation was performed in an alternative fashion by cutting and concatenating only the portions of functional time courses corresponding to the task blocks, and the classification analysis yielded similar results.

An accuracy of 0.75 (against a chance-level accuracy of 0.125 ) was obtained with a leaveone-subject-out cross-validation setting. The same classification performed on FC nodal strength values led to a lower accuracy of 0.52 , showing that structure-function dependencies alone are able to well characterize both resting-state and the different task conditions and outperform a nodal measure based on functional data only.

The LDA outputs seven meaningful discriminant directions, ordered by their discriminative power, and Fig. 2A shows the projection of the data onto the first four (i.e., the LDA scores, also shown in Supplementary Fig. 2). Notably, SDI values allow to separate very well not only resting-state from task, but also among different tasks, while this separation is less obvious when LDA is performed on FC nodal strength values (Fig. 2B).

By observing the LDA weights or coefficients, we can derive which brain regions are key for the classification. Task-specific decoding networks were obtained by linear combination of the original LDA weights through task-specific modeling of the LDA scores (see Methods), such that the final maps reflect the SDI nodal decoding coefficient for a specific task of interest compared to all other tasks. These task-specific maps, shown in Fig. 3, detect the structure-function signature of each task-related state; i.e., the brain regions that mostly characterize a specific task with respect to the others, in terms of structure-function interplay. In these maps, regions with opposite signs indicate an opposite trend of the SDI towards more structure-function 
coupling (negative sign) or more structure-function decoupling (positive sign) in the task of interest, when controlling for all other regions' effects.

The bar plots in Fig. 3 indicate the percentage of each task-specific SDI signature pattern that is involved in each functional network (7 Yeo networks ${ }^{27}$ ). Different brain networks contribute to the classification of the different task-related states, and appear consistent with previous knowledge about brain functional organization.

\section{(a) Brain decoding with structure-function dependencies}

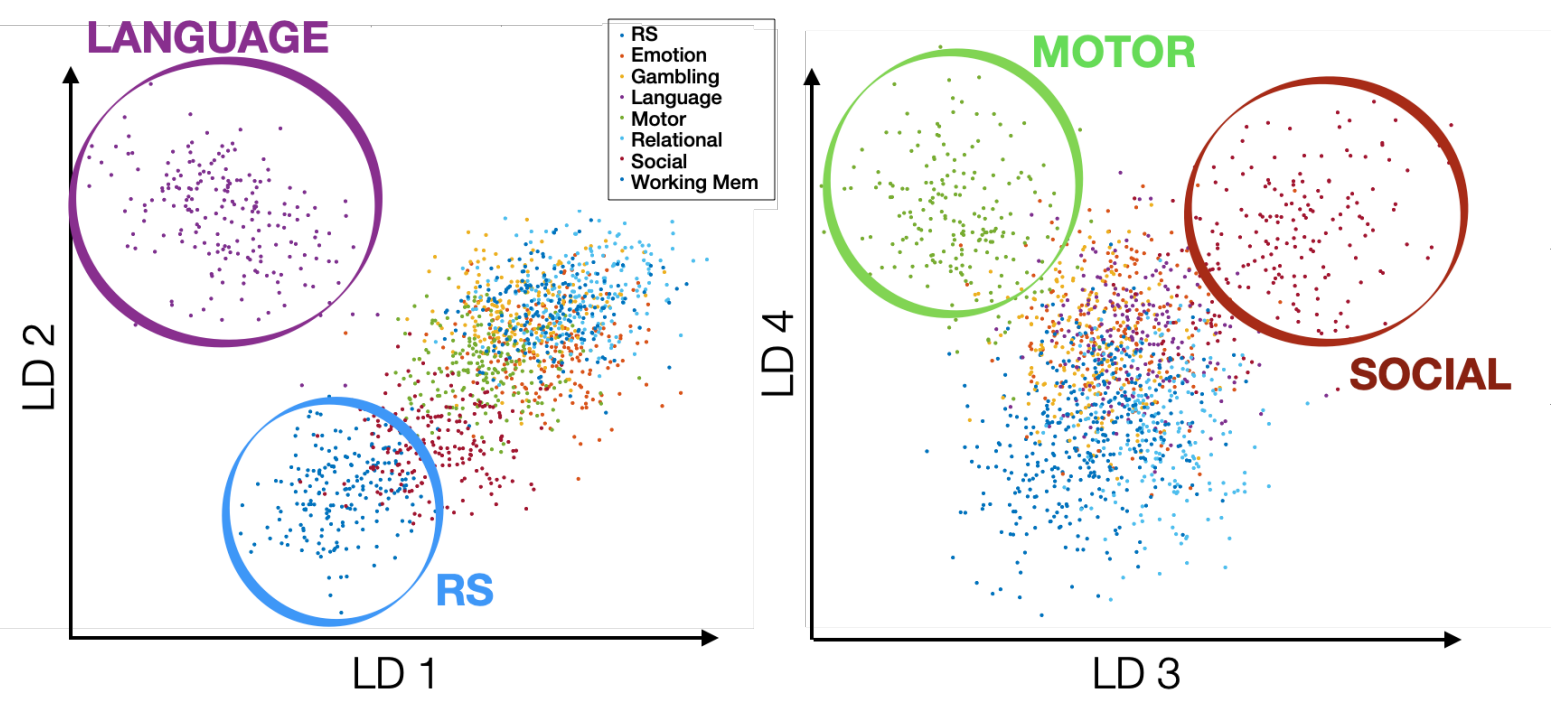

(b) Brain decoding with functional connectivity
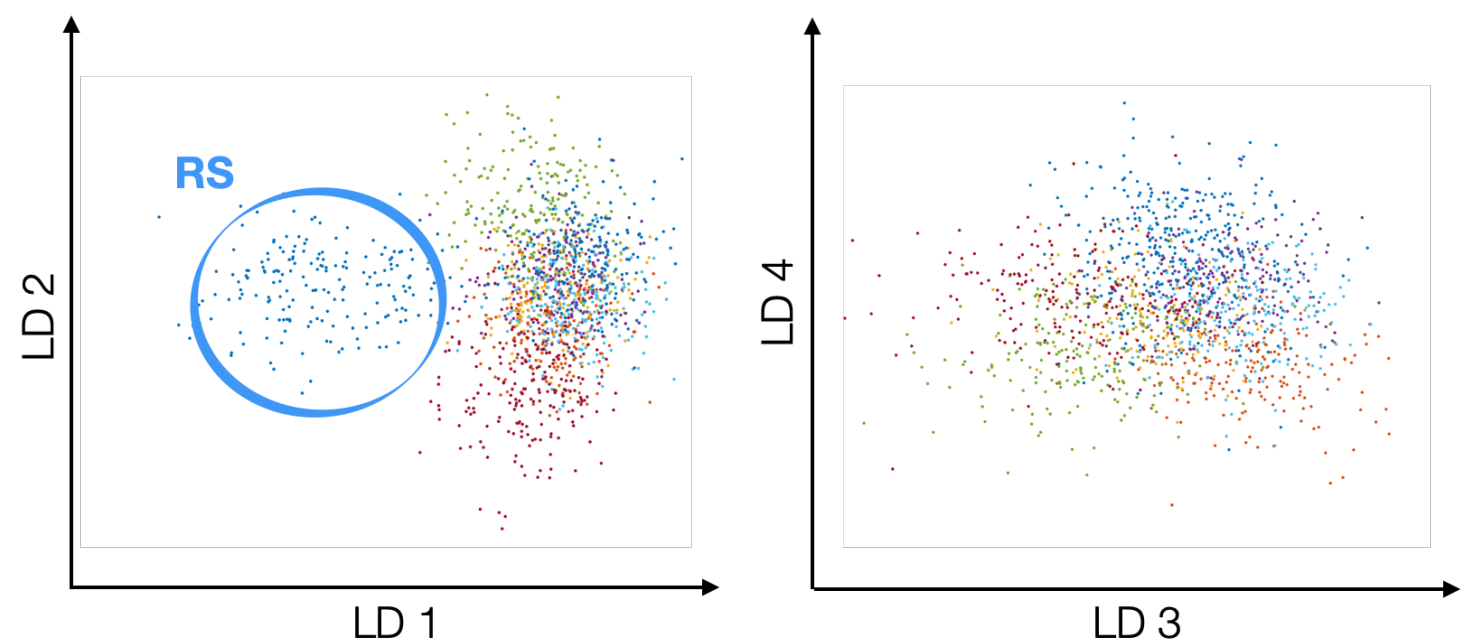

FIG. 2 - Brain decoding - Projection of the data onto the first 4 LDA discriminant directions, for (A) Structural-Decoupling Index and (B) Functional Connectivity node strength values. The higher performance of brain decoding based on structure-function interplay (accuracy $=0.75$ ) vs. functional connectivity (accuracy $=0.52$ ) is visually remarkable by the data projection in the LDA reduced space: different tasks are much better separated in $(A)$ with respect to $(B)$, which discriminates more successfully only resting-state from all other tasks. RS=resting-state; Working Mem=Working Memory. 


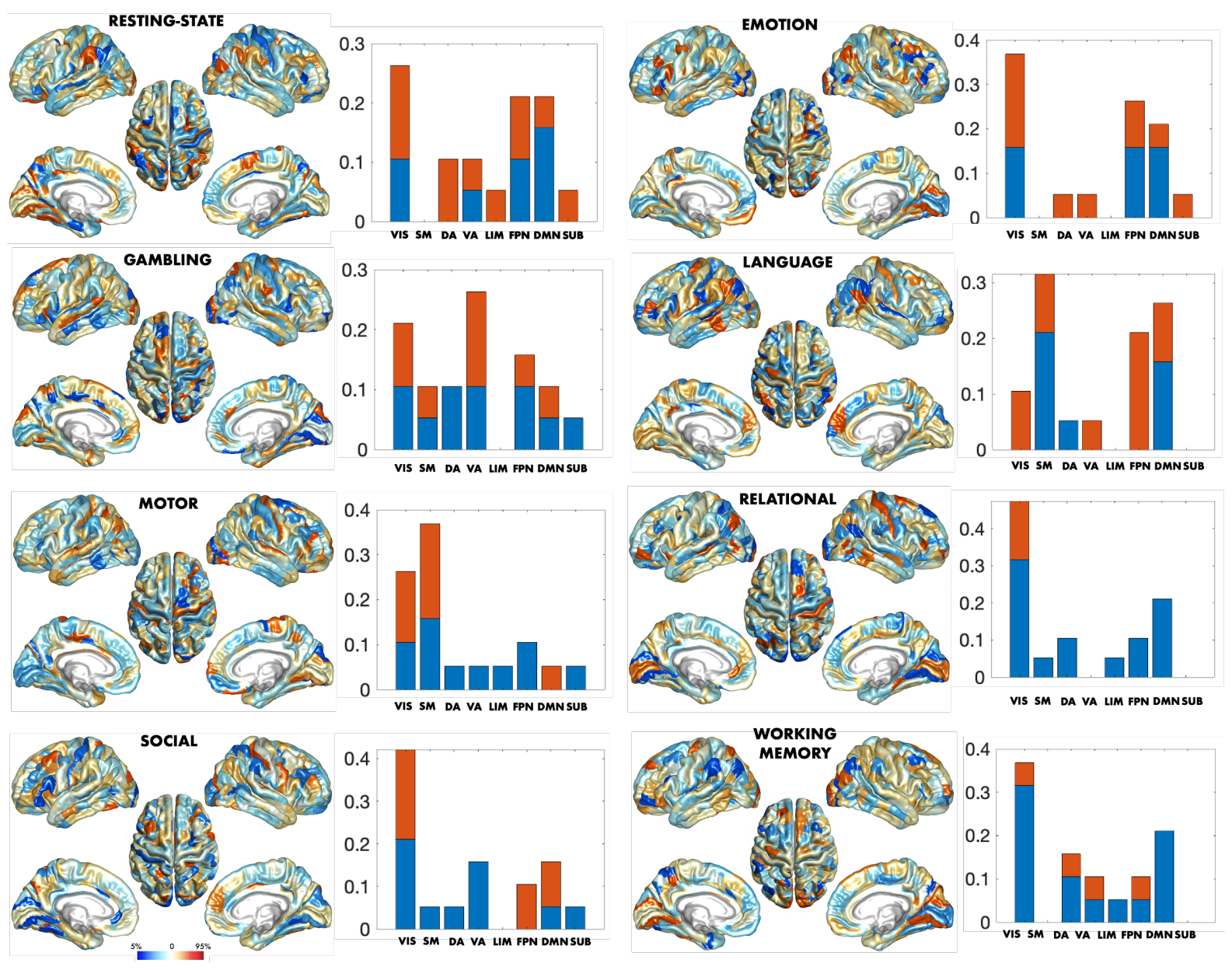

FIG. 3 - Task-specific decoding networks - For each task-related state, a brain map of most discriminative nodes is created. Regions with highest absolute value are the ones contributing the most to the classification of the specific task of interest. In a region with positive (negative) weight, we will have an increase (decrease) of the SDI during the considered task, when controlling for all other regions' effect. For each map, the bar plot on the right shows the percentage of the SDI signature pattern (i.e., of the regions with absolute value higher than the 95th percentile) belonging to known functional networks (seven Yeo functional networks and subcortical regions), distinguishing between positive (red) and negative (blue) contributions. VIS=visual; $S M=$ somatomotor; $D A=d o r s a l$ attention; $V A=$ ventral attention; LIM=limbic; FPN=fronto-parietal; $D M N=$ default mode; SUB=subcortical networks.

\section{Structure-function interplay for brain fingerprinting}

In addition to characterizing different task-related states, structure-function dependencies revealed to be highly specific to different individuals, allowing for the identification of subjects with an accuracy of 1 (both in a leave-one-subject's-task-out and in an 8-fold cross-validation setting), slightly higher than the performance of FC nodal strength values for the same classifications (0.98, for both settings). Subject identification was more difficult, but still successful, when attempting to identify individuals based on SDI patterns of only one task from SDI patterns of another (all task combinations explored). Results are reported in Table 1a, compared to the same classifications performed on FC nodal strengths (Table 1b). In most 
cases, the SDI capability of classifying subjects was higher than FC, and in particular when predicting from resting-state SDI values (used as training set) to each of the other tasks' (used as test set, one by one).

(a) Fingerprinting based on Structure-Function Dependency TEST SET

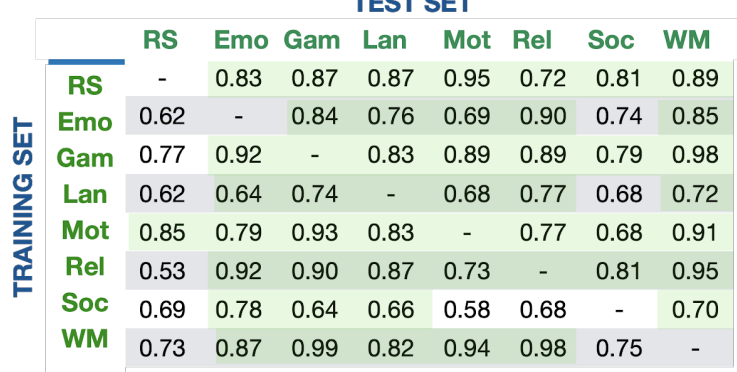

(b) Fingerprinting based on Functional Connectivity

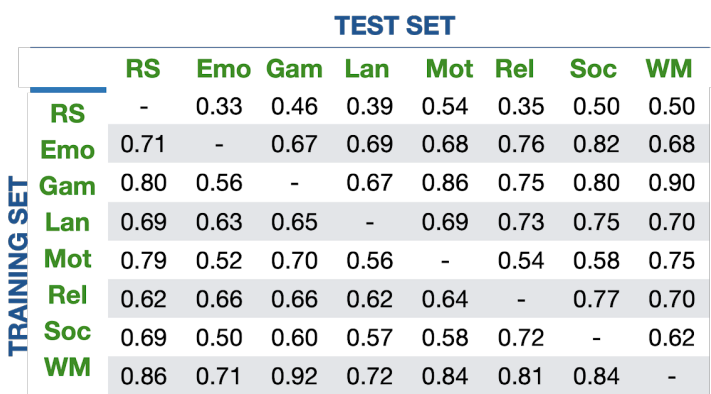

Table 1 - Brain fingerprinting - Subject classification accuracies when using only one condition (task or resting-state) for training and one for testing (all pairwise combinations), for (a) SDI and (b) FC nodal strengths. In (a), the accuracies that are found to be higher for SDI with respect to $\mathrm{FC}$ are highlighted in green.

\section{Correlation between structure-function interplay and individual cognitive traits}

Finally, structure-function interactions during resting-state appear to explain inter-individual variations of cognitive traits. Multivariate correlations between subject-specific resting-state SDI values and 10 scores measuring cognitive subdomains were assessed with Partial Least Squares Correlation (PLSC) analysis. PLSC identifies linear combinations of SDI values that maximally covary with linear combinations of cognitive scores. This analysis revealed a significant multivariate pattern ( $p<.05$ Bonferroni-corrected), with resting-state SDI values explaining $18 \%$ of the inter-individual variance of cognitive scores. In particular, SDI values in regions belonging to lower-order networks as well as to the default mode and fronto-parietal networks specifically related to fluid intelligence, spatial orientation, sustained visual attention and verbal episodic memory performances, as shown by the SDI and cognitive saliences that weigh the contribution of individual variables to the overall multivariate pattern (Fig. 4a,b). The SDI spatial pattern relating to cognitive traits (Fig. 4a) share similarities both with the SDI fingerprinting pattern (Fig. 1b), in fronto-parietal and default mode regions (rank correlation $\boldsymbol{\rho}$ $\left.=0.23, p<10^{-5}\right)$, and with the SDI decoding pattern (Fig. 1a), particularly in somatomotor areas $\left(\rho=0.50, p<10^{-5}\right)$ (Fig. 4c), but not with the SDI maps specific to the single tasks (shown in Fig. 3; results detailed in Supplementary Table 1). No significant multivariate correlation pattern was found between structure-function dependencies during tasks and cognitive traits (Supplementary Table 2). 
(a) Structure-function dependency salience

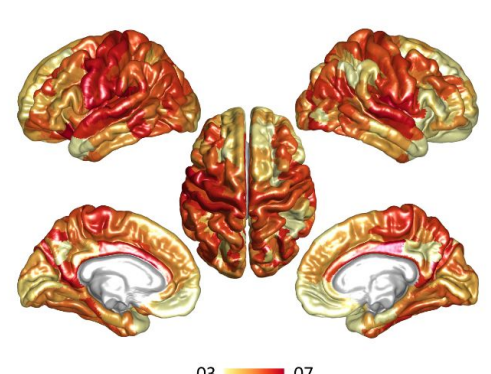

(b)

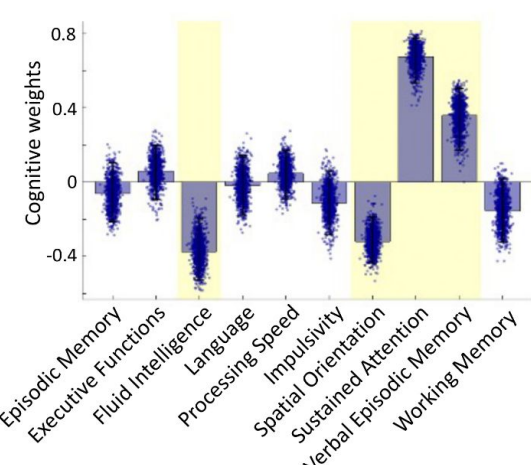

(c) Repartition among

functional networks

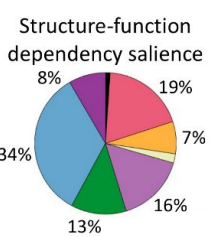

$13 \%$

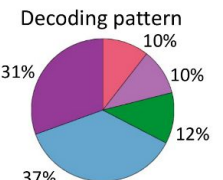

VIS SM DA VA

FPN DN

FIG. 4 - Correlation between structure-function interplay during rest and cognitive traits - Cross-subject multivariate correlation pattern between resting-state nodal SDI values (structure-function dependency salience shown in (a)) and cognitive traits (cognitive salience, average (bars) and dispersion (single dots) over 1000 bootstraps with replacement, shown in (b); cognitive subdomains with reliable nonzero salience weights are highlighted in yellow). (c) Percentage of the top 75-percentile regions in the SDI salience, SDI fingerprinting and SDI decoding patterns belonging to the seven Yeo functional networks. The cortical plots of the SDI fingerprinting and decoding patterns are shown in Fig. 1.

\section{Discussion}

Functional neuroimaging data provided measures of activity and connectivity with the ability to identify individual subjects in a group, as well as to predict brain states in relation to task execution ${ }^{2,4,8}$. In parallel, brain morphology ${ }^{12}$ and structural connectivity ${ }^{10,13}$ revealed as well the capability of uniquely identifying individuals. However, brain function and structure are conventionally considered separately and the potential of structure-function interplay in subject identification (brain fingerprinting) and state prediction (brain decoding) remains unexplored.

In relation to the first, given the high reliability of both structural and functional brain features in brain fingerprinting, we could expect structure-function dependency profiles to also uniquely characterize individuals. In line with this hypothesis, a recent study showed that the extent of alignment between structure and function correlates with individual differences in cognitive flexibility ${ }^{24}$. Instead, concerning the latter, even if we can assume brain structure will not change across different task-related states in the same individual, the way brain function couples to the underlying structure is likely to adapt to the demands of the task. In line with this, task-related functional activity was shown to be well predicted from structure only in selected brain regions, different for each task ${ }^{28}$. However, how does this structure-function relationship depend on external stimulation, cognitive engagement, and affective state and if this can be useful to decode different brain-states is still an open question ${ }^{29}$.

With these premises, we expanded here previous research by identifying the fingerprinting and decoding potential of structure-function dependencies. Specifically, the structural 
decoupling index revealed able to identify individual subjects in a group with near-perfect accuracy, indicating that the pattern of structure-function coupling is an intrinsic feature (or fingerprint) of an individual's brain organization. Further, this fingerprint appears robust to brain state changes, since even a stringent cross-validation setting with pairwise cross-task predictions delivers high fingerprinting accuracies. Nonetheless, our work also proves for the first time that structure-function interplay can predict brain states with high accuracy. Therefore, while it is true that a strong structure-function brain fingerprint exists independently from the task during which brain function is measured, structure-function dependencies are also sufficiently different across tasks to allow a reliable decoding of brain states.

The idea of a 'deep' functional fingerprint independent from brain state configuration is consistent with recent works reporting good cross-task subject identification from FC data $6,8,30$ and moderate state-dependency (compared to high subject-dependency) of functional networks ${ }^{31}$. Here, we demonstrate that the way brain function aligns (or disaligns) with the underlying structural connectivity provides additional clues on this functional brain fingerprint. We show that regional structure-function dependencies outperform a nodal measure based on FC alone in subject identification, which highlights the importance of including structural information for better fingerprinting. While brain fingerprinting does in general benefit from multimodal data integration ${ }^{32}$, structure-function interplay constitutes an interpretable and informative brain dimension delivering a compact fingerprint of brain functional organization, when compared to less parsimonious representations based on single voxels or connections 9,32

SDI also outperformed FC nodal strength when decoding task-related states with accuracies of 0.75 and 0.52 , respectively. It is important to remark here that, having regressed out task paradigms, brain decoding is based on differences in brain states driven by the task, but not "artificially" induced by the paradigm. This prevents from including any bias related to the paradigm timing that could amplify the variability between tasks and facilitate the decoding. Recent studies have shown that the cortical macro-scale gradient of structure-function coupling found at rest, opposing primary sensory and association cortices ${ }^{25,33}$, can be retrieved from task data as well ${ }^{28,34}$, suggesting similar coupling patterns both in intrinsic (rest) and extrinsic brain states. We can indeed observe the same, when comparing average SDI patterns (across subjects) among task conditions. Nonetheless, specific and non-trivial differences across tasks, captured by the brain maps shown in Fig. 3, and not clearly visible at the population level, exist and allow very accurate task-decoding.

Contributions of brain regions to subject and task identification are in fact not uniformly distributed across the cortex: two clearly distinct networks were highlighted, one for brain fingerprinting and one for task decoding (see Fig. 1).

The decoding pattern mainly involved lower-level primary sensory regions, such as somatomotor and visual networks, where structure-function coupling appears stronger both in rest and task. However, the changes in SDI that relate to the discrimination of single tasks are not obvious and show involvement of complex patterns of increase/decrease in structurefunction alignment, characterizing different tasks (Figure 3a). Notably, these SDI signature profiles suggested larger variations in structure-function interplay in functional networks known to be relevant for the considered task, for example an increased involvement of somatomotor networks during motor task, or of left temporal areas during language task (Figure 3b). 
The fingerprinting pattern, instead, shows that individual uniqueness of structure-function dependencies is mainly expressed in parietal, dorsolateral prefrontal and association cortices including the visual association and supplementary motor areas. This involves transmodal association cortices including the fronto-parietal network, which have been reported to contribute to subject identification from functional connectivity ${ }^{8}$. However, this pattern is broader and includes both regions that are coupled and regions that are decoupled with structure.

Differently from previous work that mainly focused on individual cognitive domains, here we explored multivariate correlations between structure-function dependencies and multiple cognitive traits. We show that inter-individual variations of resting-state structure-function interplay explain traits of complex cognition (fluid intelligence, spatial orientation), executive function (sustained attention) and episodic memory ${ }^{35}$, resembling descriptions of a general intelligence $\mathrm{g}$ factor previously associated with functional connectivity of the default mode network ${ }^{36}$. In particular, a relatively stronger structure-function dependency was associated with better complex cognition, recalling previous works demonstrating a link between less liberal structure-function alignment during task switching and concomitant cognitive flexibility performances ${ }^{24}$. Nonetheless, in our analyses the relationship between structure-function dependency and cognitive traits was specific to resting condition, suggesting that intrinsic rather than extrinsic brain states might better reflect general cognitive abilities. Meanwhile, this observation does not exclude that the assessment of structure-function dynamics during tasks tapping specific cognitive-behavioral subdomains may enhance the prediction of related individual traits, which may be further investigated in future research.

During rest, relatively weaker structure-function dependencies were associated with better executive and memory abilities. It might be that certain brain functions, such as immediate memory, benefit from a less constrained structure-function alignment, a configuration that might predispose the individual to the integration of new information. Other functions, such as complex reasoning, may conversely benefit from more reliable and consolidated brain communication pathways, possibly expressed in a stronger structure-function alignment $24,29,37$. While speculative, these considerations and research in this direction may offer a new understanding of cognitive control mechanisms ${ }^{38}$.

The spatial pattern of structure-function dependencies relating to cognition presented similarities both with the decoding network in lower-order somatomotor and association cortices, intrinsically characterized by strong structure-function coupling ${ }^{25}$, as well as with the fingerprinting network in fronto-parietal regions, characterized by weak structure-function interplay ${ }^{25}$. Recent work showed that structural and functional connectivities present distinct patterns of inter-individual variance as they relate to cognition ${ }^{39,40}$. Intriguingly, our results extend these findings identifying in the structure-function dependency a possible link between (divergent) structural and functional connectivity patterns in predicting behavior.

This study has a number of limitations. The usage of a grey matter parcellation as opposed to a voxel-based analysis impedes a fine-grained characterization of functional territories that can vary across subjects and tasks ${ }^{41-43}$, with possible impact on the quantification of the SDI. Nevertheless, a parcellation-based approach facilitates inter-subject comparisons, improves the signal-to-noise ratio of the estimated structural and functional measures, and enables a compact representation of brain fingerprints and decoding patterns. Finally, this study does not consider time-varying aspects of structure-function dependency ${ }^{44,45}$, which are expected to provide insight particularly in relation to task decoding and cognitive control mechanisms. 
Finally, our analyses are limited to slow temporal scales accessible with fMRI. Previous studies had attempted brain fingerprinting using electrophysiological recordings ${ }^{46-48}$, but the link between faster brain dynamics and structural topology remains poorly understood ${ }^{49,50}$. Future research may address how the hierarchy of structure-function dependencies vary at faster temporal scales, possibly carrying distinct fingerprinting and decoding information.

In conclusion, this work demonstrates that the structure-function dependencies represent a prominent signature of individual brains' organization with cognitive and behavioral consequences, while preserving task-dependent information key for brain decoding.

\section{Methods}

Data \& preprocessing. $N_{S}=100$ unrelated healthy subjects from the HCP were included in the study (ethical approval was obtained within the HCP). fMRI acquired with $N_{T}=8$ different task conditions (resting-state and 7 tasks: emotion, gambling, language, motor, relation, social, working memory), each recorded with $N_{E}=2$ phase encoding directions (right-left and left-right), as well as diffusion-weighted (DW)-MRI sequences, were pre-processed with standard pipelines, in order to obtain regional functional time courses and their structural connections, based on a parcellation with $N_{R O I}=379$ regions (360 cortical areas ${ }^{51}$ and 19 subcortical ones ${ }^{52}$ ). In particular, minimally preprocessed data from the HCP were selected 26,52 and the following additional preprocessing steps were performed. Nuisance signals were removed from voxel fMRI time courses (linear and quadratic trends, six motion parameters and their first derivatives, average white matter and cerebrospinal fluid signal and their first derivatives) and average time courses were computed in each region of the parcellation, previously resampled to the functional resolution, and z-scored. To remove the effect of the paradigm on task data, only for task classification, paradigms were regressed out trial by trial from functional time courses. Functional connectomes were obtained as Pearson's correlation between pairwise time courses and FC nodal strength was computed for each region as the sum of absolute values of all region's connections.

The same DW-MRI processing pipeline detailed in ${ }^{25}$ was used to reconstruct whole brain tractograms including 2 million fibers, using a spherical deconvolution approach and the Spherical-deconvolution Informed Filtering of Tractograms 2 (SIFT2 ${ }^{53}$ ) (https://www.mrtrix.org/). Structural connectomes were then obtained, after resampling of the same parcellation to diffusion space, as the number of tracts connecting two regions, normalized by the sum of the two regions' volumes.

SDI computation. The graph signal processing framework detailed in ${ }^{25}$ was used to obtain the SDI, for each subject and acquisition. In brief, the average SC across the population is decomposed into structural harmonics $u_{k}$ by eigendecomposition of the SC Laplacian $L=I-$ $A_{\text {symm }}$ (given the identity matrix $I$ and the symmetrically normalized adjacency matrix $A_{\text {symm }}$ of the SC):

$$
L U=U \Lambda
$$

where each eigenvalue $[\Lambda]_{k, k}=\lambda_{k}$ can be interpreted as spatial frequency of the corresponding structural harmonic (eigenvector) $u_{k}$. Functional data at each timepoint $s_{t}$ is 
then projected onto the structural harmonics by assessing spectral coefficients $\widehat{s_{t}}=U^{T} s_{t}$, and filtered into two components with ideal low- and high-pass filters, where the cut-off was set to $c=50$ spectral components for all acquisitions, to avoid task-biases. This yielded a lowfrequency functional activity component $s_{t}{ }^{C}=U^{(l o w)} U^{T} s_{t}$, which is coupled to the structure, and a high-frequency one $s_{t}^{D}=U^{(\text {high) }} U^{T} s_{t}$, more decoupled (where $U^{(\text {low })}$ and $U^{(\text {high) }}$ are $N_{R O I} \times N_{R O I}$ matrices with the $c$ first eigenvectors complemented by zeros, and with $c$ first columns of zeros followed by the $N_{R O I}-c$ last eigenvectors, respectively). The norm across time of $s_{t}{ }^{C}$ and $s_{t}{ }^{D}$ yields a general measure of coupling and decoupling for each node, and the ratio between the two corresponds to the structural-decoupling index.

Brain decoding and fingerprinting networks. A two-factors (subject and task) ANOVA on regional SDI values was performed to identify brain patterns of task and subject main effects (decoding and fingerprinting patterns, respectively; significant F-values with $p<.05$, accounting for Bonferroni correction across regions).

Brain decoding: task classification. A linear discriminant analysis with $N_{B S}=8$ classes was performed to classify a brain state $b s\left(b s=1, \ldots, N_{B S}\right.$, i.e. resting-state or one of the 7 tasks) based on the $N_{R O I} \times N_{E} \cdot N_{B S} \cdot N_{S}$ feature matrix $X$ of $S D I$ patterns for all subjects and acquisitions. LDA identifies $N_{B S}-1$ meaningful discriminative directions, each associated to a $N_{R O I^{-}}$dimensional vector of weights, which identifies brain regions mostly contributing to the classification in that LDA direction. The projection of original data $X$ onto the $N_{R O I} \times N_{B S}-1$ matrix $W$ of weights leads to the $N_{E} \cdot N_{B S} \cdot N_{S} \times N_{B S}-1$ LDA scores $L D$ :

$$
L D=X \cdot W,
$$

which maximize the inter-class variability while minimizing the intra-class one, and are used for the classification. A leave-one-subject-out (100-fold) cross-validation was implemented, where the $N_{E} \cdot N_{B S}$ acquisitions from one subject were excluded for each fold.

Task-specific decoding networks. In order to identify which brain regions mostly contribute to the specific classification of one particular task, the $N_{B S}-1$ vectors of LDA scores were considered as regressors of a linear model to predict a boxcar function $\boldsymbol{y}_{\boldsymbol{b s}}$ representing the specific task-related brain state $b s\left(\boldsymbol{y}_{\boldsymbol{b s}}=1\right.$ in the $N_{E} \cdot N_{S}$ occurrences related to task $b s$, and $y_{b s}=0$ elsewhere):

$$
y_{b s}=A \cdot \beta_{b s}+\epsilon_{b s}
$$

where the $N_{E} \cdot N_{B S} \cdot N_{S} \times N_{B S}$ design matrix $A$ contains the $N_{B S}-1$ vectors of $L D$ in addition to a baseline vector, and $\epsilon_{b s}$ represents the model error. The linear model weights $\beta_{b s}$ were then used as coefficients in a linear combination of LDA weights $W$ :

$$
w_{b s}=W \cdot \beta_{b s}
$$

yielding the task-specific weights $w_{b s}$, which represent SDI regional contribution to the classification of task bs vs. all others. A non-parametric null distribution of $99^{\prime} 999$ surrogates for $w_{b s}$ was obtained through permutation of the LDA task labelings. Standardized scores for $w_{b s}$ with respect to the null distribution were computed and used for visualization. The whole 
procedure was repeated for each task $b s$. To identify which functional networks are involved in SDI task-specific patterns, the percentage of the top 95-percentile regions in the standardized weights $w_{b s}$ belonging to the seven Yeo functional networks was computed.

Brain fingerprinting: subject classification. A second LDA with $N_{S}=100$ classes was performed to classify individuals, based on SDI values of all acquisitions. Different classification settings were explored: (1) classification of a subject $s$ doing a specific task $b s$, based on all other tasks/individuals. This was implemented with a leave-one-subject's-taskout (800-fold) cross-validation, where the $N_{E}$ entries (two different encoding directions) of subject $s$ doing task $b s$ were excluded for each fold; (2) classification of a subject $s$ doing a specific task $b s$, based on the entries related to all subjects during the remaining $N_{B S}-1$ tasks; i.e., a leave-one-task-out (8-fold) cross-validation where all entries from task $b s$ are excluded at every fold; (3) classification of a subject $s$ doing a specific task $b s$, from entries related to only one other task (all combinations explored).

SDI correlation with cognition. A Partial Least Squares Correlation analysis ${ }^{54}$ was performed to assess the presence of multivariate correlation patterns between $N_{R O I}$ nodal SDI values and 10 cognitive scores across subjects. For the cognitive scores, the 10 cognitive subdomains tested in the HCP were considered, namely, episodic memory, executive functions, fluid intelligence, language, processing speed, self-regulation/impulsivity, spatial orientation, sustained visual attention, verbal episodic memory and working memory ${ }^{55}$. For subdomains for which more than one unadjusted raw score was available, a single score was obtained by data projection onto the first component from a principal component analysis (Supplementary Fig. 3). PLSC was repeated $N_{B S}$ times, each time considering only SDI values obtained during one task $b s$. Given the dimensionality of the data, each PLSC analysis output 10 pairs of SDI-cognitive saliences (left and right singular vectors of the data covariance matrix), 10 singular values (indicating the amount of explained covariance), and 10 SDI and cognitive latent scores (data projections onto the SDI and cognitive saliences, respectively). Statistical significance of multivariate correlations was assessed with permutation testing (1000 permutations) and applying Bonferroni correction for testing 10 singular values (deemed significant for $p<.05 / 10){ }^{56,57}$. Reliability of nonzero salience values was assessed with bootstrapping procedure (1000 random samples with replacement) and computing standard scores with respect to the bootstrap distributions (salience values were considered reliable for absolute standard score > 3) ${ }^{56,57}$. The r-squared between latent scores was used to quantify the amount of cognitive traits' variance explained by the SDI values.

\section{Acknowledgments}

Data were provided by the Human Connectome Project, WU-Minn Consortium (Principal Investigators: David Van Essen and Kamil Ugurbil; 1U54MH091657) funded by the $16 \mathrm{NIH}$ Institutes and Centers that support the NIH Blueprint for Neuroscience Research; and by the McDonnell Center for Systems Neuroscience at Washington University. MGP was supported by the CIBM Center for Biomedical Imaging, a Swiss research center of excellence founded and supported by Lausanne University Hospital (CHUV), University of Lausanne (UNIL), Ecole polytechnique fédérale de Lausanne (EPFL), University of Geneva (UNIGE) and Geneva University Hospitals (HUG). EA acknowledges financial support from the SNSF Ambizione 
project "Fingerprinting the brain: network science to extract features of cognition, behavior and dysfunction" (grant number PZ00P2_185716). RL was supported by the National Centre of Competence in Research - Evolving Language (grant number 51NF40_180888)

\section{Code availability}

The code to implement this analysis will be available upon acceptance at The code to implement this analysis will be available upon acceptance at git@github.com:agriffa/LDA_SDI.git.

\section{Author contributions}

EA, MGP and RL preprocessed the data; AG, MGP and DVDV conceptualized the study; AG and MGP designed the framework and performed the analyses; all authors interpreted the results and wrote the manuscript.

\section{Competing financial interests}

The authors declare no competing financial interests.

\section{References}

1. Gao, Y., Zhang, Y., Cao, Z., Guo, X. \& Zhang, J. Decoding Brain States From fMRI Signals by Using Unsupervised Domain Adaptation. IEEE Journal of Biomedical and Health Informatics 24, 1677-1685 (2020).

2. Haynes, J.-D. \& Rees, G. Decoding mental states from brain activity in humans. Nature Reviews Neuroscience 7, 523-534 (2006).

3. Li, H. \& Fan, Y. Interpretable, highly accurate brain decoding of subtly distinct brain states from functional MRI using intrinsic functional networks and long short-term memory recurrent neural networks. Neurolmage 202, 116059 (2019).

4. Richiardi, J., Eryilmaz, H., Schwartz, S., Vuilleumier, P. \& Van De Ville, D. Decoding brain states from fMRI connectivity graphs. Neurolmage 56, 616-626 (2011).

5. Wang, X. et al. Decoding and mapping task states of the human brain via deep learning. Human Brain Mapping 41, 1505-1519 (2020).

6. Amico, E. \& Goñi, J. The quest for identifiability in human functional connectomes. Sci 
Rep 8, 1-14 (2018).

7. Biazoli, C. E. J. et al. Commentary: Functional connectome fingerprint: identifying individuals using patterns of brain connectivity. Front. Hum. Neurosci. 11, (2017).

8. Finn, E. S. et al. Functional connectome fingerprinting: identifying individuals using patterns of brain connectivity. Nature Neuroscience 18, 1664-1671 (2015).

9. Mansour L, S., Tian, Y., Yeo, B. T. T., Cropley, V. \& Zalesky, A. High-resolution connectomic fingerprints: Mapping neural identity and behavior. Neurolmage 229, 117695 (2021).

10. Kumar, K., Desrosiers, C., Siddiqi, K., Colliot, O. \& Toews, M. Fiberprint: A subject fingerprint based on sparse code pooling for white matter fiber analysis. Neurolmage 158, 242-259 (2017).

11. Valizadeh, S. A., Liem, F., Mérillat, S., Hänggi, J. \& Jäncke, L. Identification of individual subjects on the basis of their brain anatomical features. Scientific Reports 8, 5611 (2018).

12. Wachinger, C., Golland, P., Kremen, W., Fischl, B. \& Reuter, M. BrainPrint: A discriminative characterization of brain morphology. Neurolmage 109, 232-248 (2015).

13. Yeh, F.-C. et al. Quantifying Differences and Similarities in Whole-Brain White Matter Architecture Using Local Connectome Fingerprints. PLOS Computational Biology 12, e1005203 (2016).

14. Lin, Y.-C., Baete, S. H., Wang, X. \& Boada, F. E. Mapping brain-behavior networks using functional and structural connectome fingerprinting in the HCP dataset. Brain and Behavior n/a, e01647 (2020).

15. Amico, E. \& Goñi, J. Mapping hybrid functional-structural connectivity traits in the human connectome. Network Neuroscience 2, 306-322 (2018).

16. Goñi, J. et al. Resting-brain functional connectivity predicted by analytic measures of network communication. PNAS 111, 833-838 (2014).

17. Honey, C. J. et al. Predicting human resting-state functional connectivity from structural connectivity. PNAS 106, 2035-2040 (2009). 
18. Mišić, B. et al. Network-Level Structure-Function Relationships in Human Neocortex. Cereb Cortex 26, 3285-3296 (2016).

19. Avena-Koenigsberger, A., Misic, B. \& Sporns, O. Communication dynamics in complex brain networks. Nat Rev Neurosci 19, 17-33 (2018).

20. Deco, G., Jirsa, V. K. \& McIntosh, A. R. Emerging concepts for the dynamical organization of resting-state activity in the brain. Nat Rev Neurosci 12, 43-56 (2011).

21. Mišić, B. et al. Cooperative and Competitive Spreading Dynamics on the Human Connectome. Neuron 86, 1518-1529 (2015).

22. Seguin, C., Tian, Y. \& Zalesky, A. Network communication models improve the behavioral and functional predictive utility of the human structural connectome. Network Neuroscience 4, 980-1006 (2020).

23. Huang, W. et al. A Graph Signal Processing Perspective on Functional Brain Imaging. Proceedings of the IEEE 106, 868-885 (2018).

24. Medaglia, J. D. et al. Functional alignment with anatomical networks is associated with cognitive flexibility. Nature Human Behaviour 2, 156-164 (2018).

25. Preti, M. G. \& Van De Ville, D. Decoupling of brain function from structure reveals regional behavioral specialization in humans. Nature Communications 10, 4747 (2019).

26. Van Essen, D. C. et al. The WU-Minn Human Connectome Project: An overview. Neurolmage 80, 62-79 (2013).

27. Yeo, B. T. et al. The organization of the human cerebral cortex estimated by intrinsic functional connectivity. J Neurophysiol 106, 1125-1165 (2011).

28. Wu, D. et al. Hierarchy of Connectivity-Function Relationship of the Human Cortex Revealed through Predicting Activity across Functional Domains. Cerebral Cortex 30, $4607-4616(2020)$.

29. Suárez, L. E., Markello, R. D., Betzel, R. F. \& Misic, B. Linking Structure and Function in Macroscale Brain Networks. Trends in Cognitive Sciences 24, 302-315 (2020).

30. Abbas, K. et al. GEFF: Graph embedding for functional fingerprinting. Neurolmage 221, $117181(2020)$. 
31. Gratton, C. et al. Functional Brain Networks Are Dominated by Stable Group and Individual Factors, Not Cognitive or Daily Variation. Neuron 98, 439-452.e5 (2018).

32. Kumar, K., Toews, M., Chauvin, L., Colliot, O. \& Desrosiers, C. Multi-modal brain fingerprinting: A manifold approximation based framework. Neurolmage 183, 212-226 (2018).

33. Vázquez-Rodríguez, B. et al. Gradients of structure-function tethering across neocortex. PNAS 116, 21219-21227 (2019).

34. Baum, G. L. et al. Development of structure-function coupling in human brain networks during youth. PNAS 117, 771-778 (2020).

35. Moore, T. M., Reise, S. P., Gur, R. E., Hakonarson, H. \& Gur, R. C. Psychometric Properties of the Penn Computerized Neurocognitive Battery. Neuropsychology 29, 235-246 (2015).

36. Smith, S. M. et al. A positive-negative mode of population covariation links brain connectivity, demographics and behavior. Nature Neuroscience 18, 1565-1567 (2015).

37. Finn, E. S. et al. Can brain state be manipulated to emphasize individual differences in functional connectivity? Neurolmage 160, 140-151 (2017).

38. Lerman-Sinkoff, D. B. et al. Multimodal neural correlates of cognitive control in the Human Connectome Project. Neurolmage 163, 41-54 (2017).

39. Rasero, J., Sentis, A. I., Yeh, F.-C. \& Verstynen, T. Integrating across neuroimaging modalities boosts prediction accuracy of cognitive ability. PLOS Computational Biology 17, e1008347 (2021).

40. Zimmermann, J., Griffiths, J. D. \& McIntosh, A. R. Unique Mapping of Structural and Functional Connectivity on Cognition. J. Neurosci. 38, 9658-9667 (2018).

41. Laumann, T. O. et al. Functional System and Areal Organization of a Highly Sampled Individual Human Brain. Neuron 87, 657-670 (2015).

42. Salehi, M. et al. There is no single functional atlas even for a single individual: Functional parcel definitions change with task. Neurolmage 208, 116366 (2020).

43. Wang, D. et al. Parcellating cortical functional networks in individuals. Nature 
Neuroscience 18, 1853-1860 (2015).

44. Fukushima, M. et al. Structure-function relationships during segregated and integrated network states of human brain functional connectivity. Brain Struct Funct 223, 10911106 (2018).

45. Cabral, J., Kringelbach, M. L. \& Deco, G. Functional connectivity dynamically evolves on multiple time-scales over a static structural connectome: Models and mechanisms. Neurolmage 160, 84-96 (2017).

46. Fraschini, M., Hillebrand, A., Demuru, M., Didaci, L. \& Marcialis, G. L. An EEG-Based Biometric System Using Eigenvector Centrality in Resting State Brain Networks. IEEE Signal Processing Letters 22, 666-670 (2015).

47. Marcel, S. \& Millan, J. D. R. Person Authentication Using Brainwaves (EEG) and Maximum A Posteriori Model Adaptation. IEEE Transactions on Pattern Analysis and Machine Intelligence 29, 743-752 (2007).

48. Sareen, E. et al. Exploring MEG brain fingerprints: evaluation, pitfalls and interpretations. bioRxiv 2021.02.15.431253 (2021) doi:10.1101/2021.02.15.431253.

49. Finger, H. et al. Modeling of Large-Scale Functional Brain Networks Based on Structural Connectivity from DTI: Comparison with EEG Derived Phase Coupling Networks and Evaluation of Alternative Methods along the Modeling Path. PLOS Computational Biology 12, e1005025 (2016).

50. Glomb, K. et al. Connectome spectral analysis to track EEG task dynamics on a subsecond scale. Neurolmage 221, 117137 (2020).

51. Glasser, M. F. et al. A multi-modal parcellation of human cerebral cortex. Nature 536, $171-178$ (2016).

52. Glasser, M. F. et al. The minimal preprocessing pipelines for the Human Connectome Project. Neurolmage 80, 105-124 (2013).

53. Smith, R. E., Tournier, J.-D., Calamante, F. \& Connelly, A. SIFT2: Enabling dense quantitative assessment of brain white matter connectivity using streamlines tractography. Neurolmage 119, 338-351 (2015). 
54. Krishnan, A., Williams, L. J., Mclntosh, A. R. \& Abdi, H. Partial Least Squares (PLS) methods for neuroimaging: A tutorial and review. Neurolmage 56, 455-475 (2011).

55. Barch, D. M. et al. Function in the human connectome: Task-fMRI and individual differences in behavior. Neurolmage 80, 169-189 (2013).

56. Zöller, D. et al. Disentangling resting-state BOLD variability and PCC functional connectivity in 22q11.2 deletion syndrome. Neurolmage 149, 85-97 (2017).

57. McIntosh, A. R. \& Lobaugh, N. J. Partial least squares analysis of neuroimaging data: applications and advances. Neurolmage 23, S250-S263 (2004). 